\title{
Los grabados históricos en las Peñas Chicas. La anexión de la Puna de Atacama desde una estancia de Antofagasta de la Sierra (Catamarca, Argentina)
}

\author{
María Soledad Martinez y Víctor Ataliva \\ Recibido 26 de agosto 2019. Aceptado 22 de noviembre 2019
}

\begin{abstract}
RESUMEN
Las investigaciones que abordaron la anexión, hacia comienzos del siglo XX, de la Puna de Atacama al Estado argentino centraron sus indagaciones a partir de la producción documental generada por viajeros, expediciones y la burocracia administrativa. Estos aportes fueron claves para caracterizar los procesos sociales y la incidencia del Estado en un territorio concebido como marginal y localizado en los confines -geográficos y subjetivos- de la nacionalidad. A partir del análisis de unos grabados rupestres en una estancia de Antofagasta de la Sierra (Catamarca), abordamos la anexión de la puna centrándonos en las evidencias materiales. Desde una mirada arqueológica, y en particular desde las representaciones rupestres, sugerimos que las marcas inscriptas en los farallones de ignimbrita y asociados a la estancia dan indicios sobre la nueva situación política y administrativa en la que las familias puneñas desarrollaron su presente social hace más de un siglo.
\end{abstract}

Palabras clave: Grabados rupestres; Puna de Atacama; Estancia; Siglo XX.

\begin{abstract}
THE HISTORICAL ENGRAVINGS IN THE PEÑAS CHICAS: THE ANNEXATION OF THE ATACAMA PUNA FROM A ESTANCIA IN ANTOFAGASTA DE LA SIERRA, CATAMARCA, ARGENTINA. Research that addressed the annexation of the Atacama Puna by the Argentinian State in the early twentieth century has focused inquiry on the documentary archive produced by travelers, expeditions, and the administrative bureaucracy. This research was central to the characterization of the social processes and state impact over a territory conceived of as marginal and located in the confines -both geographical and subjective- of the nation. Taking the analysis of rock art engravings located in an estancia in Antofagasta de la Sierra, Catamarca, as a starting point, this paper instead approaches the annexation of the Puna from the material evidence. From an archaeological point of view, and from the rock art representations in particular, we suggest that markings inscribed in the ignimbrite walls and associated with the estancia, provide evidence of the new political and administrative situation in which the families from the Puna developed their social present more than a century ago.
\end{abstract}

Keywords: Rock engravings; Atacama Puna; Estancia; Twentieth century.

María Soledad Martinez. Instituto de Arqueología y Museo (IAM), Universidad Nacional de Tucumán (UNT). San Martín 1545, Tucumán, Argentina. E-mail: solemartinez216@hotmail.com

Víctor Ataliva. Instituto Superior de Estudios Sociales (ISES, UNT-Consejo Nacional de Investigaciones Científicas y Técnicas -CONICET-), San Lorenzo 429, Tucumán, Argentina. E-mail: vataliva@ises.org.ar

Intersecciones en Antropología 21(1), enero-junio: 99-111. 2020. ISSN-e 1850-373X

https://doi.org/10.37176/iea.21.1.2020.533

Facultad de Ciencias Sociales - UNICEN - Argentina 


\section{INTRODUCCIÓN}

Durante el último tercio del siglo XIX, una extensa área se constituyó en el centro de una disputa internacional: Chile y Argentina incorporaron una parte de la Puna de Atacama -de Bolivia, hasta $1884-$ a sus territorios. Dichas inclusiones se efectivizarían a partir de una conflagración bélica (entre Bolivia-Perú y Chile, 1879-1884), un reconocimiento consensuado (entre Bolivia y Argentina) y mediaciones diplomáticas (entre Argentina y Chile). Antes, durante y después de la anexión definitiva a la Argentina, las incorporaciones a los diferentes Estados involucraron distintas estrategias de apropiación material y simbólica de la puna (Delgado y Göbel, 2003; García y Rolandi, 2003; Haber, 2003; Karasik, 2003; Benedetti, 2005; Gil Montero, 2008; Kuperszmit, 2009; Molina Otarola, 2013; Martinez, 2016; Londoño, 2017, entre otros).

Nos proponemos contextualizar el proceso de incorporación de Antofagasta de la Sierra (en adelante, Antofagasta) al Estado argentino desde una localización específica del paisaje puneño, la estancia ${ }^{1}$ de las Peñas Chicas, y desde un primer acercamiento a una materialidad escasamente abordada en la vertiente oriental de la puna: las representaciones rupestres del siglo XX. Nos interesan, particularmente, las tres primeras décadas del siglo pasado, cuando se despliega la incorporación de la puna, considerando tal anexión como parte del proceso de expansión del Estado en el contexto de consolidación de sus fronteras. En ese marco, los grabados de la estancia, ipueden ser interpretados como marcas identitarias y referentes materiales de negociación en las relaciones entre una familia puneña y el Estado?, ¿o bien entre las familias? Pero para llegar a estas preguntas, antes debemos contextualizar el marco social y político en el que fueron generados los grabados.

\section{ANTOFAGASTA DE LA SIERRA EN CONTEXTO}

Antofagasta se encuentra en el departamento homónimo en la provincia de Catamarca (Noroeste argentino, NOA) (Figura 1). Los límites del departamento son la frontera argentino-chilena al oeste; al norte y al oriente la provincia de Salta; y al sur la cordillera de San Buenaventura. El relieve es predominantemente Ilano y sobre este se destacan las peñas columnares de ignimbrita, los volcanes y las cadenas montañosas de Calalaste y Toconquis. Es una cuenca endorreica con cauces de agua permanente a semipermanente; al Punilla, su río principal, confluyen afluentes en ambos márgenes, uno de ellos es el río Las Pitas, "arroyo de Putas" (San Román, 1896, p. 560) o "de las Puntas" (Cerri, 1993, p. 24).

Durante la Colonia, Antofagasta estuvo ligada administrativamente a la ciudad de Londres y a la gran Gobernación del Tucumán, dependiente del Virreinato del Perú. En el mundo andino, las transformaciones sociales, económicas y políticas desde el siglo XVI estuvieron modeladas por un orden propio del dominio colonial que afectó a todas las comunidades, aunque las consecuencias desestructurantes en la vida de estas fueron diferentes, según sus trayectorias históricas, sus estrategias de resistencia y los intereses coloniales específicos

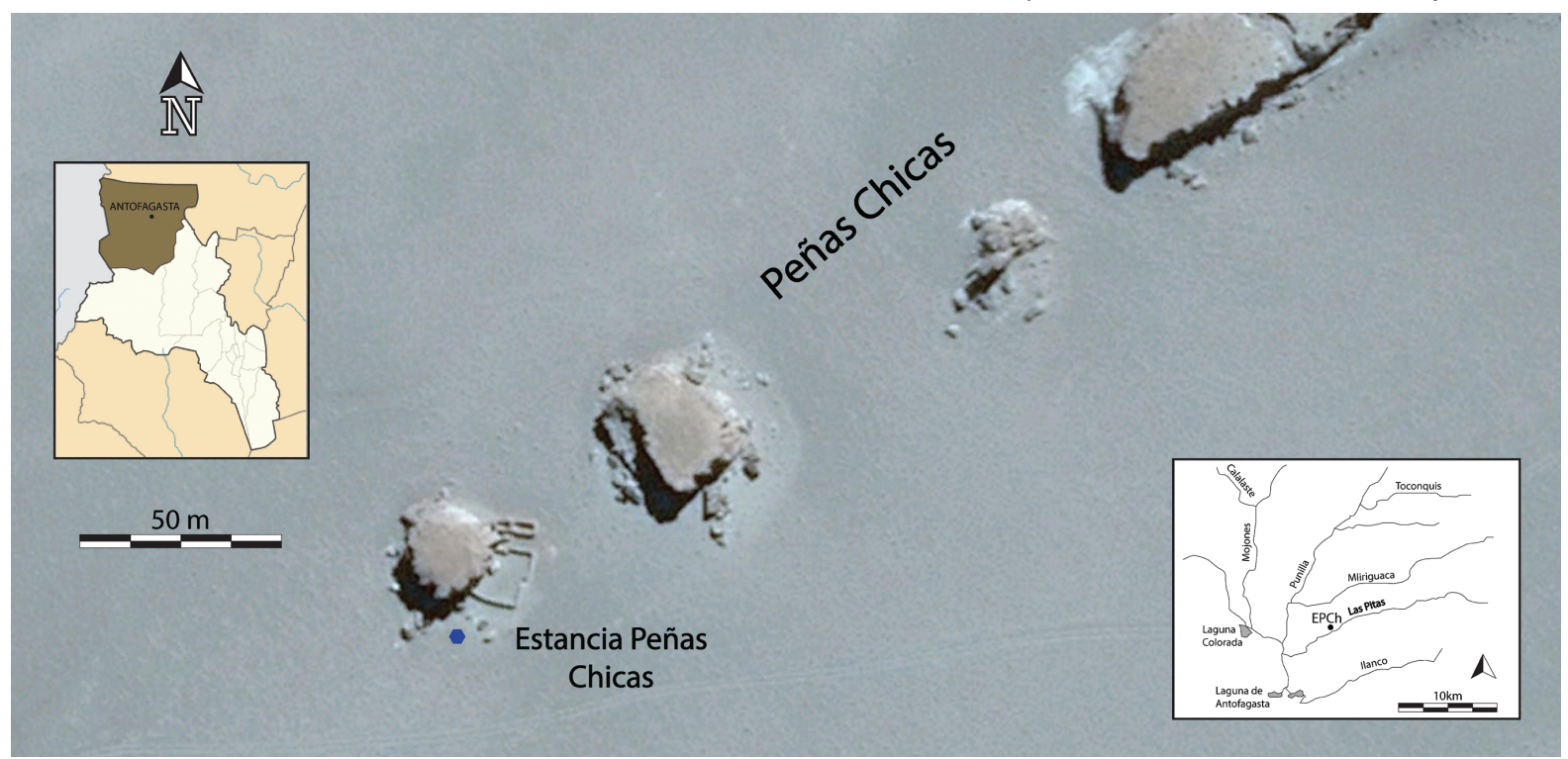

Figura 1. A la izquierda, departamento de Antofagasta de la Sierra en la provincia de Catamarca. A la derecha, el río Las Pitas en la cuenca. En el centro, la estancia en las Peñas Chicas. Fuente: imagen satelital Google Earth. 
(cf. Quiroga, 2017). En el caso de Antofagasta, en 1766 fue entregada como merced a Luis José Díaz, vecino de San Fernando de Catamarca, quien se comprometió a explotar las tierras concedidas. En 1777 se creó el Virreinato del Río de la Plata. Al dividirse, luego, en ocho unidades administrativas, Antofagasta pasaría a pertenecer a una de ellas: la Intendencia de Salta del Tucumán (García y Rolandi, 2003).

En las primeras décadas del siglo XIX se iniciaron en América del Sur los procesos emancipatorios, como consecuencia de lo cual se establecieron límites que en muchos casos no se ajustaban a las trayectorias históricas locales. Luego de las guerras independentistas, los flamantes Estados intentaron forjar la configuración de sus territorios, en función de lo cual generaron políticas tendientes a la homogenización social, biológica y cultural, uniformidad estimada como clave para la "unidad nacional" (Lenton, 2010, p. 29). Durante las últimas tres décadas del siglo XIX, el Estado argentino protagonizó sucesivas anexiones, y lo haría desde prácticas concretas: la apropiación de territorios indígenas y el sometimiento de su población (en Pampa-Patagonia y parte del Chaco), fusiones profundamente marcadas por prácticas de exterminio; por otro lado, tomó medidas tendientes a la demarcación definitiva de las fronteras interestatales.

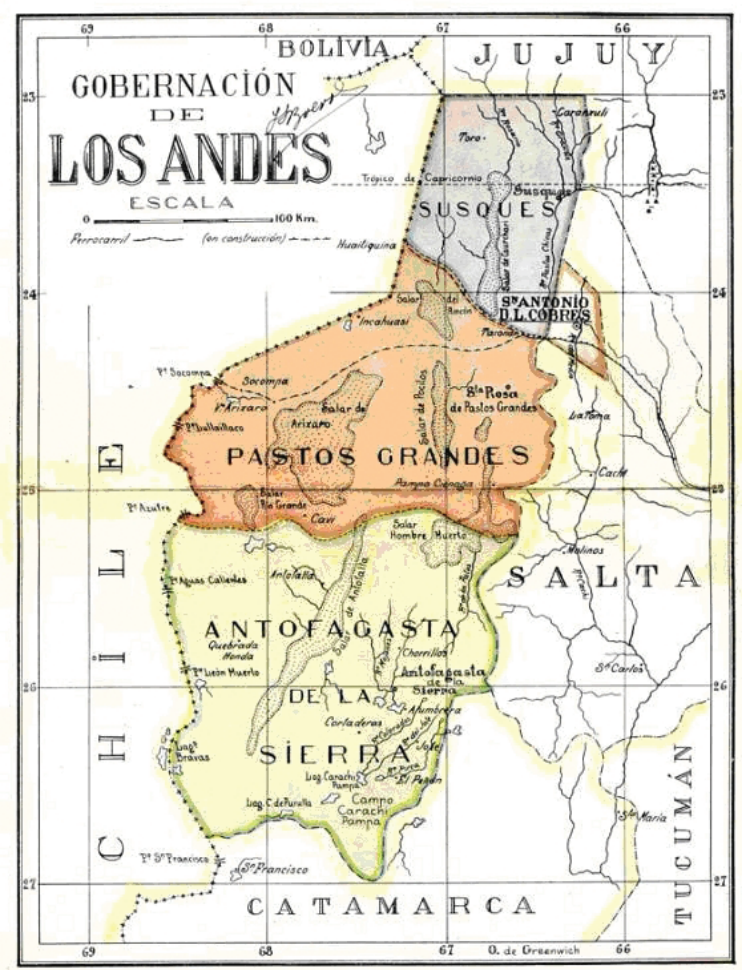

Figura 2. Territorio Nacional de Los Andes. Fuente: Jorge Boero (1941) Geografía de la Nación Argentina, Ángel Estrada, Buenos Aires (apud Benedetti, 2005, p. 266).
En efecto, en el marco de la contienda con Chile y luego del laudo arbitral (1899), por medio de la Ley 3906 del 9 de enero de 1900 se creó el Territorio Nacional de Los Andes (Figura 2), que comprendía todo el sector oriental de la Puna de Atacama que Argentina disputaba con aquel país.

En las primeras décadas del siglo XX, la región fue recorrida por viajeros y funcionarios, que centraron sus descripciones en función de intereses institucionales y saberes específicos (Benedetti, 2005). Es posible sostener que estas expediciones concibieron al paisaje y a hombres y mujeres como objetos de saber, que quedaron incorporados así a una microfísica de poder (sensu Foucault, 1998) tendiente a manipular los cuerpos y a coartar el uso autónomo del territorio (Karasik, 2003). Sin embargo, las relaciones de poder no se conforman unidireccionalmente sino que atraviesan a quienes intervienen, de manera tal que "definen puntos innumerables de enfrentamientos, focos de inestabilidad cada uno de los cuales comporta sus riesgos de conflicto, de luchas y de inversión por lo menos transitoria de las relaciones de fuerzas" (Foucault, 1998, p. 34). Por lo tanto, no solo deben enfatizarse las relaciones de poder que cada uno de los Estados puso en práctica con los puneños, ya que dejaríamos de lado la capacidad de estos para generar estrategias de reproducción social (sensu Bourdieu, 2011), colectivas y/o familiares, frente a los dispositivos de anexión y control de las sucesivas administraciones.

\section{PRIMERAS DÉCADAS DEL SIGLO XX: FAMILIAS Y SOCIEDAD}

Es posible sugerir que en Antofagasta, como en el distrito de Huáncar (Susques, Jujuy), hacia inicios de siglo XX las familias constituían los "núcleos centrales de producción económica, de distribución y consumo y de reproducción social" (Göbel, 2003, p. 203). Con una dinámica particular -con asiento en la movilidad pastoril-, cada unidad doméstica disponía de sectores de pastoreo y de instalaciones estratégicamente localizadas y empleadas en función del ciclo productivo anual, disponibilidad de recursos y decisiones familiares; ejemplo de ello es la familia de Vicente Morales y la distribución de sus asentamientos ("domicilio grande", estancia, puestos, etc.) y corrales.

Al día siguiente de la creación del territorio nacional, en una misiva dirigida al presidente Julio A. 
Roca y firmada por una veintena de hombres y mujeres (transcripta en Cerri, 1993, pp. 64-66), las familias de Antofagasta expusieron los "abusos, tropelías y desbarajustes" por el accionar de "individuos que cavilosamente tratan de apoderarse de nuestros derechos". El conflicto surgió ante la presentación de un "encargado" que comunicó que "todos los terrenos de Antofagasta pertenecen a [Isaac Aranibar y Fermin Sonete]", quien pretendía obligarlos "á pagar mil contribuciones según el reglamento que á su amaño han inventado". Ante esta situación, las familias sostuvieron: "Cosa extraña para nosotros, puesto que estos terrenos son comunales, y que durante muchos años nuestros padres y nosotros, hemos pagado tributo como tributarios" primero de Bolivia y después de Chile. Acto seguido, nombran

al antiguo tributario D. José Salvatierra para que protestara ante abusos incalificables, y así lo hizo y protestó con nosotros todos, y se acordó de mandar á su Excelencia el Presidente, para que ahora y siempre nos ampare con su justicia y benevolencia en los derechos como poseedores de nuestras propiedades.

La misiva brinda indicios sobre distintos aspectos de la sociedad y expone, con ajustada terminología, una síntesis de la situación administrativa y lo que se considera un atropello a sus "derechos", frente a lo cual las familias se reunieron, nombraron un "encargado" -con importante trayectoria en el territorio-y argumentaron con precisión. A horas de la flamante creación del territorio nacional, un grupo de familias señalaron "estar sujetos a las órdenes y disposiciones de su Excelencia a quien Dios guarde". ${ }^{2}$

Este rápido reacomodamiento en un escenario de fronteras itinerantes -después de todo, las familias no tenían motivos para suponer que desde ese momento serían argentinos y no luego, y de nuevo, chilenos o bolivianos- remite al accionar estratégico para posicionarse frente al Estado. Asimismo, expone la existencia de espacios deliberativos comunitarios $y$, coyunturalmente, de consensos para abordar situaciones que afectaban los intereses colectivos $y$, como en este caso, exponer una diligente reacción para sostener denuncias o acciones. Es decir, aquí también las familias configuraban la trama política y organizativa local.

Fue durante esta primera década del siglo XX que se implementaron una serie de dispositivos para "argentinizar" la puna: se institucionalizó la autoridad del Estado por medio de los juzgados de
Paz y la Policía, y se organizó la administración de la gobernación (Benedetti, 2005). En el caso de Antofagasta también se registra, para la etapa -aunque discontinua-, una mayor actividad educativa (Kuperszmit, 2009). Por entonces, Antofagasta estaba integrada a una compleja red macrorregional que articulaba, económica y socialmente, una diversidad de poblados de ambas vertientes de los Andes: además de los arrieros -esencialmente de ganado mular- que la atravesaban, también desde y hacia Antofagasta se proyectaban los intercambios, "cambalacheo" o trueques de una diversidad de productos requeridos por las familias de aquí y allende la cordillera (cf. García y Rolandi, 2003; Molina Otarola, 2013).

Como se desprende de los escritos de viajeros y exploradores de fines del XIX e inicios del XX, ciertas vegas -de propiedad comunal- y los alfalfares familiares daban sustento tanto a los animales arreados o que invernaban allí, como a la hacienda de las unidades domésticas. Además de esta intensa actividad, San Román había destacado las producciones familiares: "Se dá bien la alfalfa; se logra el maíz i el trigo; la hortaliza, se cultiva sin inconveniente i la papa común encuentra allí su propio clima i suelo" (San Román 1896, p. 561); mientras Sundt advertía sobre la relevancia de otras dos actividades: "El alumbre (extraído del Volcán Alumbrera) junto con cueros de vicuña i unas pocas ovejas, son los únicos artículos de exportación de los habitantes de Antofagasta. El alumbre se consume en la Arjentina para teñir i curtir" (Sundt, 1909, p. 96).

\section{GRABADOS EN CONTEXTO}

La estancia de las Peñas Chicas, como la denomina la familia Morales, constituye uno de los tantos asentamientos que se encuentran en el curso del río Las Pitas (a $3543 \mathrm{msnm}, 26^{\circ} 0158^{\prime \prime} \mathrm{S}$ y $67^{\circ}$ $213^{\prime \prime}$ W). Patrimonio de don Vicente Morales y su familia, dataría de fines de siglo XIX e inicios del siguiente. En función de un proyecto que abordó el pasado colonial y republicano de esta porción de la puna (Martinez, 2012), centramos el análisis en la arquitectura en pie y los grabados integrados a dicha estancia. A continuación, y a medida que exponemos someramente el sitio, incorporamos las interpretaciones que surgieron a partir de las entrevistas realizadas a don Vicente, las que abordaron distintos aspectos de su trayectoria personal y familiar en la estancia (Figura 3$)^{3}$ 


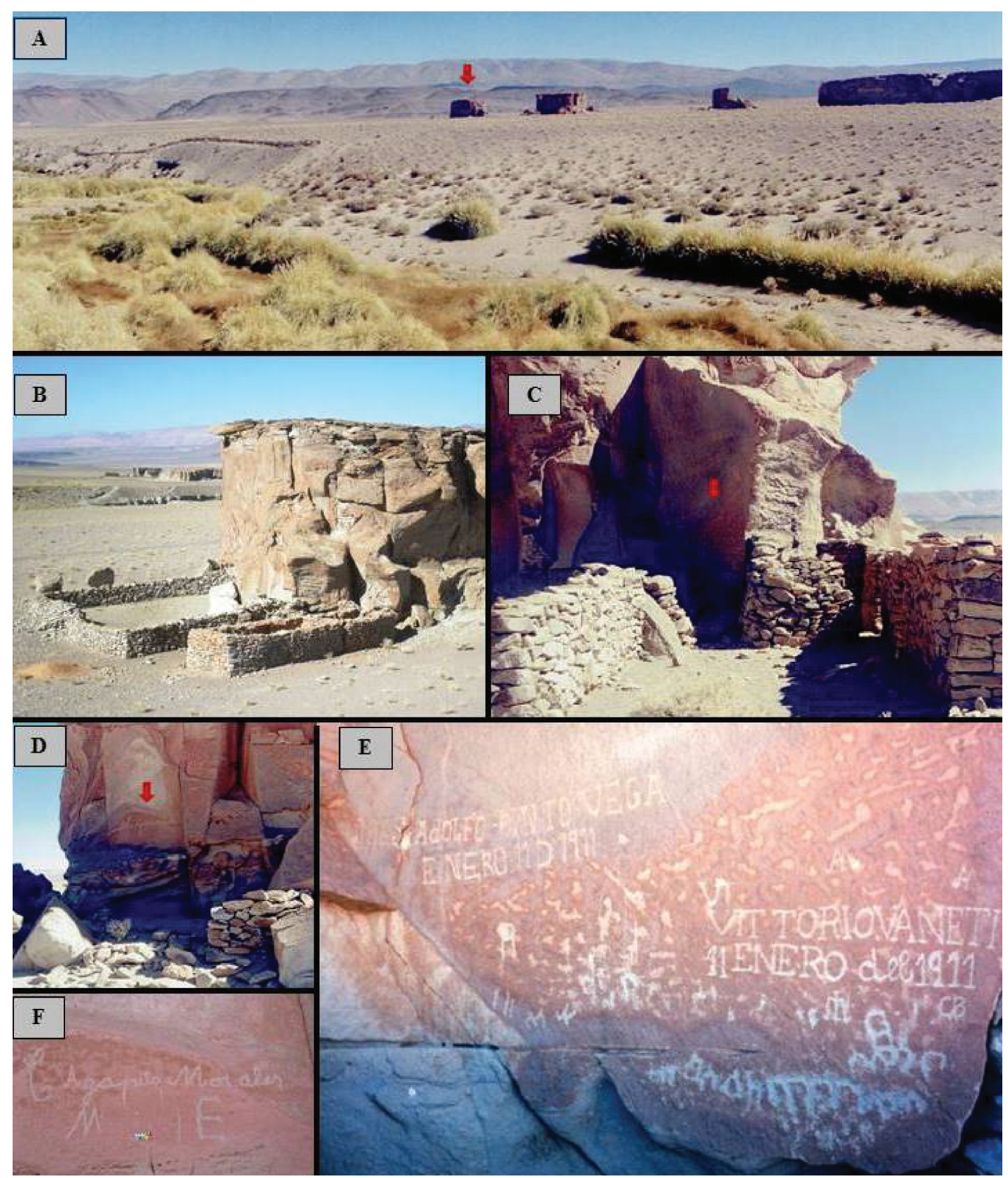

Referencias: A: La estancia en las Peñas Chicas. B: Vista de la estancia desde el noreste. C: Interior de la estancia desde el pasillo (la flecha señala los grabados). D: Chiquero y grabados. E: Grabados en la estancia (panel 1) y F: Grabados del chiquero (panel 2).

Figura 3. La estancia de las Peñas Chicas (EPCh) y los grabados en contexto.

La estancia está conformada por una diversidad de construcciones - de pirca seca- en la base de una peña columnar de ignimbrita (Figura 4). Se ingresa por el este, por un amplio pasillo que divide en dos las construcciones: hacia un lado, el corral (es decir, al sur), y hacia el otro, tres recintos y unas pequeñas estructuras (esto es, al norte del pasillo). Dos recintos rectangulares contiguos cuentan cada uno con una única abertura, en los muros sur de ambos, las que conectan con el pasillo (mientras que en los muros norte se localizan las hornacinas). En el primero de los recintos se encuentran dos poyos enfrentados y separados por el piso de la habitación. El poyo constituye un rasgo muy común en la arquitectura andina y cumple con distintas funciones; en este caso, se trata de dos plataformas 


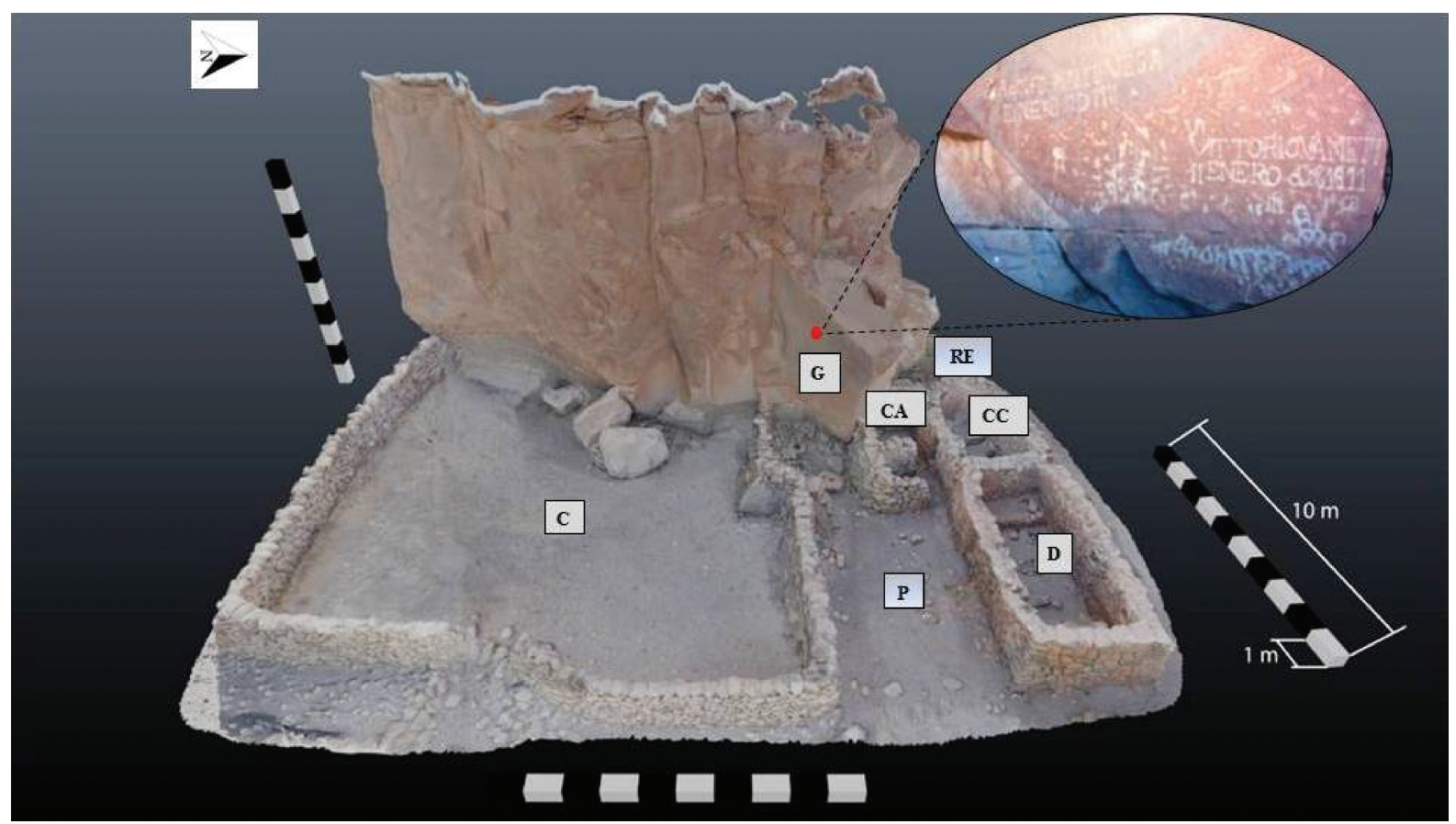

Referencias: C: Corral. P: Pasillo. D: Dormitorio. CC: Cocina cerrada. CA: Cocina abierta. G: Grabados. RE: Recinto externo.

Figura 4. La estancia de las Peñas Chicas.

macizas de ignimbrita y argamasa rojiza cuyos límites superiores están conformados por unas rocas planas, delgadas tipo lajas, seleccionadas para generar una superficie horizontal. Entre los intersticios y por debajo de estas lajas -y en algunos casos, por encima- se detecta una argamasa arcillosa (en el pasado, toda la superficie, horizontal y vertical, del poyo contaba con tal terminación). El interior del recinto también estaba cubierto por esta argamasa, cuyo fin era aislarlo térmicamente, para mitigar así los efectos del frío viento puneño. Con respecto a su función, según don Vicente: "Ha sido el dormitorio de mi finada madre y de mi papito. Nosotros dormíamos así, par a par...", señalando la manera en que se ubicaban los hermanos/as en el piso, ${ }^{4}$ dispuestos paralelamente entre los dos poyos. "Para entonces no había tantas posibilidades como ahora... El finadito mi padre sabía sobar cuero y darnos...", refiriendo al empleo de los cueros (como aislante) para acostarse sobre ellos. Un poyo, entonces, era empleado para dormir y en el otro se colocaban una diversidad de enseres, ropa, mercaderías, etc.; por lo que el recinto cumplía tanto con la función de dormitorio como de depósito.

En el centro del recinto adyacente -que cuenta con un poyo al pie del muro oeste- se registra un fogón en un rasgo circular de rocas ígneas. Este recinto, como el anterior, no conserva el techo, pero aquí funcionó la cocina cerrada. Al suroeste de esta se ubicaba la cocina abierta, donde culmina el pasillo. Otra estructura, contigua a esta cocina, cumplía con la función de pequeño depósito donde se guardaban las ollas y demás utensilios de uso doméstico. Hacia el oeste de la cocina cerrada $-y$ también al resguardo de la peña- se encuentra otra estructura que fue empleada, según don Vicente, primero como espacio de trabajo con las tinturas y el alumbre extraído de La Alumbrera (a unos 10 kilómetros al sureste), y luego como basurero (en superficie se hallaban fragmentos de cerámica y vidrio, carbones, restos faunísticos, etcétera).

Al sur del pasillo se localiza el corral (con su única abertura en el este, aunque también se accede desde el pasillo, por una roca en la que tallaron pequeños peldaños) $y$, finalmente, el panel con los grabados en el farallón, estratégicamente localizado (el pasillo desemboca en este panel).

En la estancia, entonces, confluyen el espacio residencial y el productivo (principalmente con asiento en las prácticas pecuarias), instalaciones que remiten a la economía familiar y al empleo estacional. Durante gran parte del siglo XX la estancia estuvo incorporada a un circuito familiar que incluía el "domicilio grande" en Falda de Ilanco, a unos siete kilómetros al este (las distancias están siempre referidas con respecto a la estancia). En palabras de don Vicente, se encontraba en Ilanco el "caserío más importante", "el domicilio de mis padres" y en el que se residía parte del año (de hecho, nació allí el 28 de julio de 1919). Los meses más 
cálidos (diciembre hasta abril-mayo) se trasladaban a Peñas Chicas, desde donde los niños asistían a la escuela del pueblo (a unos cinco kilómetros al sur); las mejores pasturas se hallaban en este sector, donde había, además, abundante agua. El "domicilio grande" y la estancia constituían las cabeceras de un circuito que incluía también otros corrales y puestos. ${ }^{5}$ Por ejemplo, un corral a unos 500 metros al este de la estancia; otro emplazado en el interior de un alero en altura (Punta de la Peña 4 o Alero Don Vicente), ${ }^{6}$ a unos dos kilómetros al noreste; un puesto y un corral -al pie del farallón donde se encuentra dicho alero- donde residía don Vicente hasta unos años antes de fallecer (2014); y una residencia en el pueblo de Antofagasta (donde murió). Aunque todos estos sitios son relevantes para pensar la dinámica de una familia durante el período, nos centramos aquí en la estancia y sus grabados.

\section{Marcas en las peñas}

En esta etapa de la investigación, nuestro objetivo es contextualizar las manifestaciones en el marco de la anexión de Antofagasta al Estado argentino y abordar lo definido como contexto de uso, es decir, las maneras en que las "representaciones son utilizadas como materia de significación y administradas para su observación y reproducción" (Aschero, 1996, p. 178). Estas expresiones plásticas fueron mencionadas por Podestá (1986-1987) en su trabajo de síntesis sobre el arte rupestre de Antofagasta y el sitio nominado como Peña Chica 4. Su investigación brinda información específica sobre el curso inferiormedio de Las Pitas y las representaciones prehispánicas del sitio Peña Chica 3 (40 metros al noreste). En esta peña se encuentra un pequeño chiquero de unos $6 \mathrm{~m}^{2}$, sobre el que -en el farallón- también se grabaron inscripciones. ${ }^{7}$

Respecto de las representaciones correspondientes a los períodos colonial y poscolonial, solo en las últimas tres décadas fueron abordadas de manera sistemática. Una de las contribuciones pioneras es la de Gallardo et al. (1990) en la vertiente trasandina. No realizaremos una revisión de los antecedentes que fueron conformando, desde entonces, un relevante corpus de investigaciones que abordan las manifestaciones desde el siglo XVI hasta el XX; en todo caso, las investigaciones sobre las marcas de ganado en sitios de las mesetas meridionales de Río Negro (Moldes, 1999), y en San Juan, tanto en el Parque Provincial Ischigualasto (Podestá y Rolandi, 2001; Podestá et al., 2006; Romero Villanueva 2012), como en el piedemonte de la sierra de la Huerta (Cahiza, 2010-2012) constituyen antecedentes específicos y contemporáneos a la estancia.

Una última consideración antes de describir los grabados: según don Vicente, algunas de las manifestaciones rupestres eran iniciales, por lo que las concebimos como tales. Estas son muy similares a las marcas de ganado tal como las describen Podestá et al. (2006, pp. 175-176). Según Julio Morales -nieto de don Vicente-, la familia contaba con una marca (q49), la que se colocaba en "la pierna derecha de los animales grandes, los burros y mulas", y señalaban "las cabras, ovejas y llamas,

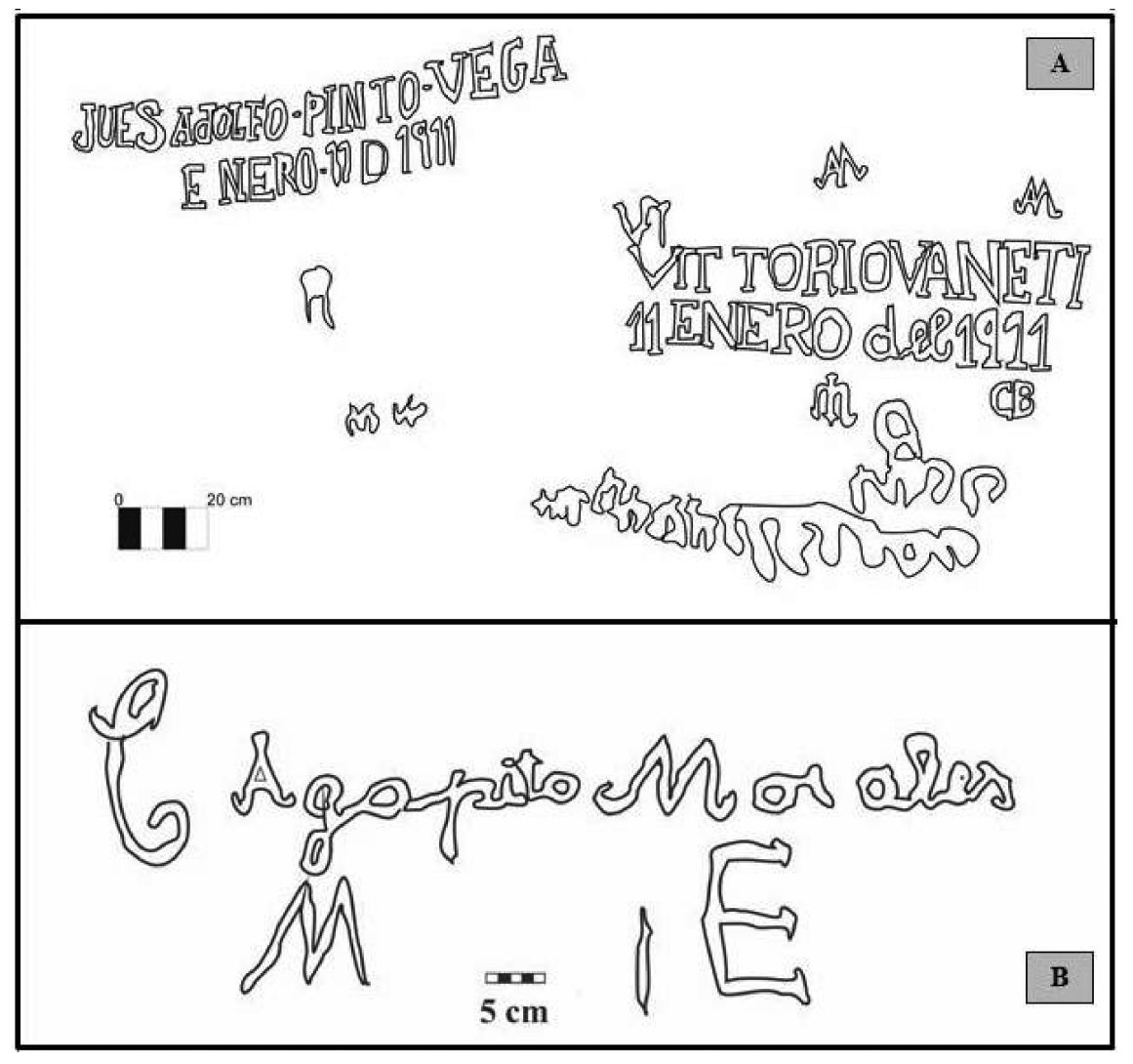

Referencias: A: Panel 1 (al final del pasillo). B: Panel 2 (en el chiquero).

Figura 5. Parte de los grabados de la estancia. 
con un corte en la punta de la oreja derecha y agujero en el medio, y en la oreja izquierda corte en forma de $L^{\prime \prime}{ }^{8}$

Ahora bien, del conjunto de representaciones, la mayoría fueron confeccionadas con la técnica de picado; excepcionalmente, en el caso de un nombre y apellido, con la técnica de rayado (Vicente Morales, y que podría ser posterior al resto de los grabados), y dos iniciales elaboradas mediante incisiones (AM). Como en los sitios anteriormente mencionados, aquí también se exponen iniciales, nombres, apellidos y fechas; sin embargo, se trata de un universo muy limitado de manifestaciones en tanto no superan la veintena (Tablas 1 y 2 ). Comenzamos la descripción con los grabados vinculados directamente a la estancia (panel 1); continuamos con otras representaciones que están en el mismo farallón; y, finalmente, con las asociadas al chiquero (panel 2) (Figura 5).

Tanto por sus disposiciones en el panel 1 como por sus tamaños, describimos primero los nombres, apellidos y fechas, para luego centrarnos en las iniciales. El primer grabado se encuentra en la parte superior izquierda del panel: se trata de inscripciones en letra tipo imprenta mayúscula (a excepción de la letra d): JUES AdOLFO-PINTO-VEGA, por debajo, la fecha ENERO-11 D 1911. En este panel, a la derecha y más abajo (con letra de imprenta mayúscula y de molde minúscula), las inscripciones: VITTORIO VANETI y 11 ENERO del 1911. Por encima de VITTORIO, las letras VI dan indicios de un primer intento de ejecución; nos preguntamos si se trata de una prueba o si quien realizó el grabado lo descartó por algún motivo (¿estético?) para reiniciar por debajo la inscripción del nombre (Tabla 1).

Con respecto a las iniciales, dos se encuentran por encima y dos por debajo de la última representación mencionada: dos motivos figurativos similares, compuestos por la combinación de las letras $\mathrm{A}$ y M; los otros dos, también figurativos, uno conformado por la combinación de las letras $\mathrm{M}$ e I y el otro por las letras C y B. También por debajo de la primera inscripción descripta (JUES...), se distinguen un motivo compuesto por la $\vee$ en el centro de una M y una $M$ invertida o $W$. Se presentan, además, otros grabados: una pequeña oquedad de la que se proyectan dos líneas paralelas y, en la parte inferior derecha del panel, un grabado que, para don Vicente, es una caravana de Ilamas.

Saliendo de la estancia y bordeando el farallón por el norte, se registran: un nombre (JOSÉ), dos iniciales (AM, realizadas posiblemente con algún

\begin{tabular}{|c|c|c|}
\hline Tipos de motivos & Leyenda completa & Observaciones \\
\hline \multirow{2}{*}{$\begin{array}{c}\text { Nombre y } \\
\text { Apellidos / Fecha }\end{array}$} & JUES AdOLFO-PINTO-VEGA & \multirow{4}{*}{$\begin{array}{c}\text { Panel incorporado a la estancia; } \\
\text { es decir, grabados en asociación } \\
\text { directa con las construcciones } \\
\text { (Panel 1). }\end{array}$} \\
\hline & ENERO-11 D 1911 & \\
\hline \multirow{2}{*}{$\begin{array}{c}\text { Nombre y } \\
\text { Apellido / Fecha }\end{array}$} & VITTORIO VANETI & \\
\hline & 11 ENERO del 1911 & \\
\hline Nombre y Apellido & Vicente Morales & \multirow{2}{*}{$\begin{array}{c}\text { Inscripciones aisladas } \\
\text { en el farallón de la estancia } \\
\text { pero cercanas a esta. }\end{array}$} \\
\hline Nombre & JOSÉ & \\
\hline Nombre y Apellido & Agapito Morales & $\begin{array}{l}\text { Panel donde se encuentra } \\
\text { el "chiquero" (Panel 2). }\end{array}$ \\
\hline
\end{tabular}
elemento punzante como un cuchillo o un clavo) y, con la técnica de rayado, Vicente Morales (letra de molde).

Mencionamos que a unos 40 metros al pie de otra peña se encuentra un chiquero. Para su confección se emplearon grandes bloques desprendidos de la peña y la técnica muraria de pirca seca. Sobre el chiquero, y en el farallón, se encuentran los graba-

Tabla 1. Nombres y apellidos.

\begin{tabular}{|c|c|c|c|c|}
\hline $\begin{array}{l}\text { Tipos de } \\
\text { motivos }\end{array}$ & $\mathbf{n}$ & Letras & Observaciones & Referente \\
\hline \multirow{9}{*}{ Marcas } & 2 & AM & $\begin{array}{l}\text { Motivo figurativo, combinación de ambas letras. } \\
\text { Panel } 1 .\end{array}$ & $\begin{array}{l}\text { Agapito } \\
\text { Morales }\end{array}$ \\
\hline & 1 & IM & $\begin{array}{l}\text { Motivo figurativo, combinación de letras (la I en } \\
\text { el centro de la M). Panel } 1 .\end{array}$ & $\begin{array}{l}\text { Ignacio } \\
\text { Morales }\end{array}$ \\
\hline & 1 & CB & $\begin{array}{l}\text { Motivo figurativo compuesto por ambas letras. } \\
\text { Panel } 1 .\end{array}$ & $\begin{array}{l}\text { Carmela } \\
\text { Vázquez }\end{array}$ \\
\hline & 1 & ¿MV? & $\begin{array}{c}\text { Motivo figurativo, combinación de ambas letras } \\
\text { (¿la } \vee \text { en el centro de la } M \text { ?). Panel } 1 .\end{array}$ & $\begin{array}{l}\text { ¿Morales y Vásquez? / } \\
\text { ¿Vásquez de Morales? }\end{array}$ \\
\hline & 1 & ¿W? & ¿W o M invertida? Panel 1. & ¿Morales? \\
\hline & 2 & AM & $\begin{array}{c}\text { Motivo figurativo, combinación de ambas letras. } \\
\text { Aislados en la peña (uno encima de otro y } \\
\text { distanciados } 5 \mathrm{~cm} \text { ). }\end{array}$ & $\begin{array}{l}\text { Agapito } \\
\text { Morales }\end{array}$ \\
\hline & 1 & $\mathrm{C}$ & Motivo figurativo con volutas. Panel 2. & Ciriaco \\
\hline & \multirow{2}{*}{1} & M & \multirow{2}{*}{$\begin{array}{l}\text { Motivo figurativo compuesto por ambas letras } \\
\text { separadas una de otra. Panel } 2 \text {. }\end{array}$} & \multirow{2}{*}{$\begin{array}{l}\text { Morales } \\
\text { Estanislao }\end{array}$} \\
\hline & & $\mathrm{E}$ & & \\
\hline
\end{tabular}

Tabla 2. Iniciales y referentes. dos: Agapito Morales; a su izquierda, la inicial C (con volutas); y por debajo del nombre y apellido, y en la misma línea horizontal pero distanciadas, las iniciales $\mathrm{M}$ y $\mathrm{E}$.

Hasta aquí, la mayoría -no la totalidad-de los grabados. Tal como recurrentemente sostuvo don Vicente, una parte de estos grabados son 
iniciales de los miembros de su familia: "están aquí las iniciales de mi finado mi hermano mayor", mientras señalaba la AM, de Agapito Morales. Por su parte, IM y CB son, respectivamente, las iniciales de su padre, Ignacio Morales, y de su madre, Carmela Vázquez. Asimismo, y sobre el chiquero, el nombre completo de su hermano mayor más las iniciales C, de Ciriaco, y ME, de Estanislao Morales (Tabla 2). Las iniciales, entonces, remiten de manera directa a referentes identificables o, si se prefiere, a identidades nominales: además de los nombres y apellidos mencionados no locales (y sobre los que estamos indagando en distintos acervos documentales), los farallones exponen las iniciales y también nombres y apellidos (José, Agapito y Vicente) de integrantes de esta familia.

\section{DISCUSIÓN}

A partir de los testimonios de don Vicente y Julio, como así también de las evidencias materiales, es posible sostener que la construcción de la estancia comenzó hacia fines de siglo XIX y fue intensamente incorporada a la dinámica familiar durante las primeras décadas de siglo XX, marco temporal en el que se desenvuelven las disputas de soberanía entre Chile y Argentina sobre el oriente de la Puna de Atacama y se concreta la incorporación definitiva al segundo país (Martinez, 2012). En este contexto, retornamos a las preguntas iniciales. Primero: ies posible abordar desde los grabados aspectos referidos a la identidad familiar, en tanto marcas que remitan a una determinada unidad doméstica? Y segundo: ¿es probable abordar las relaciones entre una familia y el Estado?, ¿o bien las relaciones entre las unidades domésticas?

Es necesario realizar una distinción en función de las identidades nominales a las que remiten los grabados: un primer grupo -mayoritario-, constituido por nombres e iniciales de integrantes de la familia Morales; un segundo grupo, conformado por aquellos referentes extrapuneños (Pinto y Vaneti).

Con respecto a la autoría del primer grupo, y como sostienen los testimonios, fueron elaborados por quienes construyeron $y / o$ habitaron la estancia. Las iniciales y los nombres de varios integrantes de la familia Morales quedaron inscriptos en las peñas: por parte de las mujeres, únicamente Carmela (y no sus hijas); por los hombres, Ignacio y sus hijos, Agapito, Vicente, Estanislao, José Santos y Ciriaco. Por lo tanto, se puede afirmar que efectivamente estas marcas remiten a la identidad de una familia y que constituyen para los Morales una evidencia contundente de la propiedad sobre la peña y su continuidad histórica en este sector de Las Pitas. De los hermanos, el mayor, Agapito (nacido en 1913) -con cinco referencias- es el que más se encuentra representado. Según don Vicente, muchos de estos grabados (del primer grupo) fueron realizados por Agapito cuando aprendió a escribir, por lo que sería posible plantear que algunas iniciales corresponderían, al menos, a la década de 1920.

Sin embargo, creemos que lo más importante es abrir la discusión sobre los destinatarios de estas marcas. Si asumimos que las iniciales tuvieron como fin señalizar la estancia, inscribir los derechos sobre esta propiedad, y que su rol fue que los observadores identificaran a sus dueños rápidamente y sin ambigüedades, entonces, ¿a quiénes van dirigidos los mensajes?

Tal como las investigaciones históricas, etnográficas y antropológicas pusieron de manifiesto, los pastores son extremadamente cuidadosos respecto del uso de los lugares comunitarios -o comunalesde aquellas construcciones que corresponden a las familias (puestos, corrales, etc.) y los límites de los sectores de pastoreo están sujetos a negociaciones no exentas de tensiones (Nielsen, 1996; Delgado y Göbel, 2003; Gil Montero, 2008, entre otros). Entonces, ¿por qué la insistencia de los Morales en remarcar su propiedad cuando sus contemporáneos la conocían? En todo caso, ¿ es posible sostener que estos mensajes fueran dirigidos a otras familias con las que disputaban este sector de Las Pitas?, ¿o los destinatarios de estas representaciones son no-puneños?

Si concordamos con de Certau (1999) ${ }^{9}$ sobre el espacio privado, las formas de exponer u ocultar en el hogar, las circulaciones permitidas y no consentidas, lo que resalta tanto por presencia como por ausencia, las restricciones y libertades de comportamiento, los límites del hacer, etc., entonces sería factible plantear algunas interpretaciones sobre las motivaciones de esta familia para marcar la estancia. En primer lugar, es importante señalar que los grabados (aunque exceptuando los cuatro alejados del panel 1) están asociados de manera directa a las construcciones. Los paneles 1 y 2 se destacan por sus disposiciones respecto de lo edificado. La amplia visibilidad -por tamaño y localización en el panel 1-de las inscripciones que no remiten a identidades locales (las únicas visibles desde el 
exterior de la estancia) contrasta con las iniciales, que solamente pueden ser observadas si se cumple, al menos, con dos prescripciones: a) estar en el interior del corral; o, b) ingresar a la estancia por el único acceso (el pasillo que desemboca en los grabados). En ambos casos, se requiere de la invitación para ingresar (salvo, claro está, cuando la familia se hallaba ausente de las Peñas Chicas). No está de más señalar la relevancia de los corrales para los pastores del NOA, que constituyen importantes espacios de sociabilidad entre las familias en ciertas épocas del año, por ejemplo, cuando se realizan señaladas, yerras, esquilas o actividades vinculadas a lo sanitario y/o reproductivo de los animales, o bien durante fechas clave del calendario ritual, como el $1^{\circ}$ de agosto. Por lo tanto, quienes se hallaban en el corral podían observar las iniciales. Y también, como destacamos, quienes ingresaban por el pasillo (con o sin autorización de los Morales).

Por lo expuesto, es posible que sean otras familias puneñas las destinatarias de lo que transmitían las marcas (es decir, lo que comunicaban insistentemente los Morales en ambas peñas). Aunque se requiere avanzar con investigaciones referidas a la tenencia y propiedad de la tierra en Antofagasta, como las realizadas para otros contextos del siglo XIX (por ejemplo, Barros, 2008; Gil Montero, 2008), y posteriores, a los fines de contar con un panorama más preciso sobre las dinámicas familiares (y sobre los posibles conflictos y disputas entre las unidades domésticas y las relaciones con las administraciones estatales, etc.), esta aproximación a los grabados contribuye a pensar las relaciones entre las familias en un contexto original, marco en el que hombres y mujeres "comenzaban" a ser argentinos y argentinas. Si las administraciones previas (la boliviana hasta el fin de la guerra, en 1884, y la chilena desde entonces y por quince años) habían ejercido cierta presencia institucional, no ocurriría lo mismo a partir de la creación del territorio nacional, cuando el Estado se propuso la "argentinización" de esta porción de la puna. Queda pendiente definir si las iniciales de los padres de don Vicente fueron realizadas por ellos mismos, por un tercero -bajo el requerimiento de ellos- o por Agapito u otro familiar.

Ahora bien, un incidente de 1910 referido por Kuperszmit (2009) podría aportar algunos indicios que guíen las futuras indagaciones respecto de la complejidad de las prácticas institucionales. Los protagonistas: por un lado, un grupo de familias, por el otro, el juez de Paz y el comisario. En un relevamiento realizado por el juez en el "pueblo de Antofagasta", detectó "cinco casas inhabitadas, con sus respectivos patios y potreros", e informó al comisario. Unos días después, este envió una misiva al gobernador del territorio:

En mi carácter de Comisario hago que dichos pobladores conserven sus derechos de dueños de las ya citadas casas, teniendo en cuenta que si bien es cierto que abandonan temporalmente sus viviendas, es porque tienen que retirarse de la población a cuidar su ganado que pastan en las vegas que S. E. se habrá dado cuenta quedan en diferentes rumbos y distancias de este Pueblo. (apud Kuperszmit, 2009, p. 50)

La relevancia de estas miradas, ajenas a las lógicas locales pero comprometidas por sus roles en Antofagasta de 1910 en tanto representan distintos niveles del poder estatal, radica en que posibilitan una aproximación a las prácticas burocráticas y haceres institucionales durante la primera década del siglo XX. Aunque sorprende el desconocimiento sobre las dinámicas puneñas -aspecto que remite al carácter foráneo de ambos funcionarios-, lo importante del incidente es que revela el interés de las administraciones por ejercer cierto tipo de control sobre las familias y sus bienes.

Desde el pueblo de Antofagasta retornamos a la estancia y nuevamente a la visibilidad de los grabados. Las identidades foráneas del panel 1 son las únicas claramente visibles desde el exterior de la estancia pero, como advertimos, las iniciales solamente pueden ser observadas desde el interior del corral y del pasillo, por lo que aquí también cabría preguntarse si, por ejemplo, los destinatarios podrían ser esos funcionarios (como el juez de Paz o el comisario del incidente) que debían comprobar, conocer o relevar los bienes de las familias puneñas.

Con respecto a lo definido -por sus identidades no locales- como segundo grupo, es decir, Pinto y Vaneti, señalemos que, por la responsabilidad de uno de ellos (juez) ${ }^{10}$ y por la fecha (11 de enero de 1911, y que no necesariamente data el evento del grabado), en futuras indagaciones abordaremos sus roles y relación con la estancia y los Morales.

Como destacamos con relación a la misiva del 10 de enero de 1900 enviada al presidente Roca, es posible sugerir que los puneños acumularon experiencias con las sucesivas administraciones, que les permitieron -a partir de la anexión a la 
Argentina- contar con capitales específicos para entablar las "nuevas" relaciones con el "nuevo" Estado al que fueron integrados. En todo caso, es factible plantear que hombres y mujeres de Antofagasta capitalizaron tales experiencias para posicionarse, con cierto margen de maniobra, frente a la "nueva" coyuntura (después de todo, esta anexión podría haber sido pensada como provisoria, en función de lo ocurrido durante las tres décadas previas). En el caso particular que abordamos, entendemos que una materialización de estas experiencias son los propios grabados, que son interpretados aquí en tanto dispositivo identitario generado por una familia a los fines de enfatizar la apropiación de este sector de Las Pitas frente a sus contemporáneos y, tal vez también, frente al Estado. Para ello, la familia Morales generó una respuesta original, al emplear una herramienta clave en la comunicación administrativa y plenamente identificada con el Estado: inscribirá -o escribirá- su presencia en las peñas con los signos alfabéticos propios de los haceres burocráticos.

\section{CONSIDERACIONES FINALES}

El marco general que sustenta las preguntas que motivaron este trabajo se inscribe en un proyecto que aborda las trayectorias políticas, económicas y sociales de las familias de Antofagasta durante el siglo XIX y las primeras del XX (Martinez, 2016), marco temporal en el que se desenvuelve la incorporación de la Puna de Atacama al Estado argentino. Estas indagaciones están ancladas en materialidades y podrían resumirse así: ¿es posible pensar desde las evidencias -rasgos arquitectónicos, textiles (cf. Martinez, 2012) o, como en este caso, grabadosaspectos que remitan a la conflictividad entre las familias y entre estas y el Estado? En otras palabras, desde el registro material, ¿es viable relevar esas instancias de tensiones y reacomodamiento que suponen la instauración de unas nuevas estructuras -políticas, administrativas, burocráticas- con las que la población local debió interactuar a inicios del siglo XX? Sostenemos que, para avanzar con tales indagaciones, se requería de un primer abordaje a la problemática desde una evidencia material que, sin ambigüedades, remitiera al período. Es por ello que centramos esta aproximación a las representaciones rupestres, las que, sugerimos, dan indicios del marco político y social en el que se desenvuelve la cotidianeidad de una familia puneña de Antofagasta de la Sierra durante el período.

\section{Agradecimientos}

A la familia Morales por la predisposición siempre amorosa con la que reciben las visitas del Equipo Antofagasta del Instituto de Arqueología y Museo (Universidad Nacional de Tucumán). A Roy Casañas Rígoli por el trabajo de fotogrametría de la estancia. A Matías Lépori por la confección del abstract. A Sara López Campeny, Victoria Isasmendi, M. Lorena Cohen y Carlos A. Aschero, con quienes -en distintos momentos- realizamos las distintas etnografías en Antofagasta.

\section{REFERENCIAS CITADAS}

Aschero, C. A. (1996). Arte y Arqueología: una visión desde la Puna argentina. Chungara, 28(1-2), 175-197.

Barros, A. (2008). Identidades y propiedades: transiciones territoriales en el siglo XIX atacameño. Estudios Atacameños, 35, 119-139.

Benedetti, A. (2005). Un territorio andino para un país pampeano Geografía histórica del Territorio de los Andes (1900-1943) [tesis doctoral, Universidad de Buenos Aires]. Repositorio institucional UBA: http:// www.filo.uba.ar/contenidos/investigacion/institutos/geo/ ptt/TesisdoctoradoBenedetti.pdf

Bourdieu, P. (2011). Las estrategias de la reproducción social. Siglo XXI.

Cahiza, P. (2010-2012). Las Piedras Marcadas. Representaciones rupestres del piedemonte occidental de la Sierra de Valle Fértil, San Juan. Anales de Arqueología y Etnología, 65-67, 121-135.

Cerri, D. (1993). El Territorio de Los Andes. Reseña Geográfica Descriptiva. Universidad Nacional de Jujuy (original publicado en 1903).

de Certau, M. (1999). La invención de lo cotidiano 2: habitar, cocinar. Universidad Iberoamericana.

Delgado, F. y Göbel, B. (2003). Departamento de Susques: la historia olvidada de la Puna de Atacama. En A. Benedetti (Comp.), Puna de Atacama (Sociedad, economía y frontera) (pp. 81-104). Alción Editora.

Foucault, M. (1998). Vigilar y castigar. Nacimiento de la prisión. Siglo XXI.

Gallardo, F., Castro, V. y Miranda, P. (1990). Jinetes sagrados en el desierto de Atacama: un estudio de arte rupestre andino. Boletín del Museo Chileno de Arte Precolombino, 4, 27-56.

García, S. y Rolandi, D. (2003). Antofagasta de la Sierra, Provincia de Catamarca. Su historia en los documentos y la tradición oral. En A. Benedetti (Comp.), Puna de Atacama (Sociedad, economía y frontera) (pp. 137197). Alción Editora 
Gil Montero, R. (2008). La construcción de Argentina y Bolivia en los Andes Meridionales. Población, tierras y ambiente en el siglo XIX. Prometeo.

Göbel, B. (2003). "Llegamos a un pueblo de indios abandonado". Aportes de la expedición sueca a la etnografía de la Puna de Atacama. Pacarina, 3, 143-158.

Goñi, R. (1995). El uso actual de los aleros: algunas implicancias arqueológicas. Cuadernos del Instituto Nacional de Antropología, 16, 329-341.

Haber, A. (2003). ¿Cómo, cuándo y por qué la Puna se convirtió en texto? Pacarina, 3, 169-178.

Karasik, G. (2003). La etnografía en el cuerpo: dominación e insumisión en las exploraciones de principios del siglo XX en Jujuy (Argentina). Pacarina, 3, 179-192.

Kuperszmit, N. G. (2009). Relaciones políticas, sociales y económicas en un pueblo de pastores. Antofagasta de la Sierra, Territorio Nacional de los Andes, 1900-1943 [tesis de licenciatura, Universidad de Buenos Aires]. Repositorio institucional UBA: http://repositorio.filo.uba. ar/handle/filodigital/890

Lenton, D. (2010). La "cuestión de los indios" y el genocidio en los tiempos de Roca: sus repercusiones en la prensa y la política. En O. Bayer (Coord.), Historia de la crueldad argentina (pp. 29-50). El Tugurio.

Londoño, W. (2017). La puna de atacama y el problema de la otredad: una mirada a la primera mitad del siglo XX. Estudios Atacameños, 55, 219-230.

Martinez, M. S. (2012). Prácticas textiles resignificadas en contextos de transformaciones en Antofagasta de la Sierra (Provincia de Catamarca-Argentina). Integrando materialidad del pasado y la oralidad del presente [tesis de grado inédita, Universidad Nacional de Tucumán].

Martinez, M. S. (2016). Estrategias ante un nuevo orden social. Transformaciones y permanencias en Antofagasta de la Sierra, Catamarca. Serie Monográfica y Didáctica, 54, 411-416.

Moldes, B. (1999). Perduración del simbolismo aborigen en el espacio rionegrino (1884-1920). Actas del XII Congreso Nacional de Arqueología Argentina 2: 445-450.

Molina Otarola, R. (2013). Cordillera de Atacama: movilidad, frontera y articulaciones collas-atacameñas. En A. Núñez, R. Sánchez y F. Arenas (Eds.), Fronteras en movimiento e imaginarios geográficos. La cordillera de Los Andes como espacialidad sociocultural (pp. 189220). RIL editores.

Nielsen, A. (1996). Competencia territorial y riqueza pastoril en una comunidad del sur de los Andes
Centrales (Dpto. Potosí, Bolivia). Zooarqueología de Camélidos, 2, 67-90.

Podestá, M. M. (1986-1987). Arte Rupestre en Asentamientos de Cazadores-Recolectores y Agroalfareros en la Puna Sur Argentina: Antofagasta de la Sierra, Catamarca. Relaciones de la Sociedad Argentina de Antropología, XVII(1), 241-263.

Podestá, M. M. y Rolandi, D. (2001). Marcas en el desierto. Arrieros en Ischigualasto (San Juan, Argentina). Boletín de la Sociedad de Investigación del Arte Rupestre de Bolivia, 15, 63-73.

Podestá, M. M., Rolandi, D., Re, A., Falchi, M. P. y Damiani, O. (2006). Arrieros y marcas de ganado: expresiones del arte rupestre de momentos históricos en el desierto de Ischigualasto. En D. Fiore y M. M. Podestá (Eds.), Tramas en la Piedra. Producción y Usos del Arte Rupestre (pp. 169-190). World Archaeological Congress (WAC); Sociedad Argentina de Antropología (SAA); Asociación Amigos del Instituto Nacional de Antropología (AINA).

Quiroga, L. (2017). Entramados rebeldes de puna y valles en el Tucumán (siglo XVII). Valle de Londres, provincia de los Diaguitas. Una perspectiva cartográfica. Estudios Atacameños, 55, 203-218.

Romero Villanueva, G. (2012). Saliendo del anonimato. Los arrieros por su nombre. Análisis de iniciales y nombres grabados en el desierto de Ischigualasto (provincia de San Juan). En N. Kuperszmit, T. Lagos Mármol, L. Mucciolo y M. Sacchi (Eds.), Entre pasados y presentes III. Estudios contemporáneos en Ciencias Antropológicas (pp. 585-603). Mnemosyne.

San Román, F. (1896). Desierto i Cordillera de Atacama. Tomo Segundo. Jeografía. Imprenta Nacional de Santiago de Chile.

Sundt, L. (1909). Estudios jeológicos i topograficos del desierto i la puna de Atacama. Sociedad Nacional de Minería de Santiago de Chile.

\section{NOTAS}

1.- Advertimos que cuando en este trabajo referimos a la categoría estancia no remitimos a su extensión territorial, sino a la unidad de análisis conformada por una diversidad de construcciones y funciones vinculadas a la vida cotidiana en el mundo pastoril y que adquieren características propias (arquitectónicas y de uso temporal o estacional, etc.) en función del rol (o roles) que cumple(n) en la dinámica diseñada por las familias puneñas.

2.- Lo entrecomillado son citas y palabras textuales que corresponden a la misiva transcripta por Cerri (1993, pp. 64-66).

3.- Esta sección incorpora las entrevistas realizadas a don Vicente en abril de 2003, junto a Sara M. L. López Campeny y a cargo del coautor; en febrero de 2009, a cargo de la 
coautora y Victoria Isasmendi; y nuevamente en diciembre de 2011, a cargo de la coautora junto a Lorena Cohen y Carlos A. Aschero.

4.- Don Vicente tuvo dos hermanas, Petrona y Jesús del Milagro, y cinco hermanos, Estanislao, Ciriaco, Nicolás, José Santos y Agapito.

5.- En este caso -como en otros contextos andinos-, los puestos de la familia Morales consisten en instalaciones asociadas a las prácticas de manejo animal y de características constructivas similares a las de la estancia aunque con una superficie mucho menor; por ejemplo, en ocasiones se trata tan solo de un recinto adosado o muy cercano al corral.

6.- Es relevante señalar que algunas investigaciones etnoarqueológicas y etnográficas en distintos paisajes y con diferentes formaciones sociales correspondientes al siglo XX pusieron en manifiesto que los aleros y cuevas "no son utilizados permanentemente o usualmente" y exponen "una baja redundancia de uso" (Goñi, 1995, p. 331). En este caso en particular, la familia Morales instaló un corral -que dejó de ser empleado como tal al menos hace cuatro décadas- bajo el reparo del alero. Hacia mediados de 2001, y como consecuencia de las intensas Iluvias que habían dañado el techo del puesto donde residía don Vicente al pie del farallón, el coautor de este texto pudo observar que trasladaba sus puyos para dormir en el alero. Lo citamos como un ejemplo referido al uso transitorio del alero en función de coyunturas específicas.

7.- En el NOA el término remite, al menos, a dos acepciones: 1) espacio delimitado con una pirca, un cerco vivo, etc., o un corral de madera, para ubicar allí vacunos, camélidos, etc., recién nacidos o de pocos meses; y, 2) espacio donde se encuentran los porcinos. Don Vicente refiere a la primera acepción.

8.- Julio Morales, entrevista de la coautora, febrero de 2017.

9.- Teniendo presente que sus reflexiones están sustentadas en experiencias y contextos urbanos europeos.

10.- Que no se encuentra en el listado de jueces que se desempeñaron en Antofagasta (Kuperszmit, 2009). 
M. S. Martinez y V. Ataliva 